\title{
Dynamics of particle-particle collisions in a viscous liquid
}

\author{
F.-L. Yang and M. L. Hunt ${ }^{a)}$ \\ Division of Engineering and Applied Sciences, California Institute of Technology, \\ Pasadena, California 91125
}

(Received 15 May 2006; accepted 13 September 2006; published online 8 December 2006)

\begin{abstract}
When two solid spheres collide in a liquid, the dynamic collision process is slowed by viscous dissipation and the increased pressure in the interparticle gap as compared with dry collisions. This paper investigates liquid-immersed head-on and oblique collisions, which complements previously investigated particle-on-wall immersed collisions. By defining the normal from the line of centers at contact, the experimental findings support the decomposition of an oblique collision into its normal and tangential components of motion. The normal relative particle motion is characterized by an effective coefficient of restitution and a binary Stokes number with a correlation that follows the particle-wall results. The tangential motion is described by a collision model using a normal coefficient of restitution and a friction coefficient that are modified for the liquid effects.
\end{abstract}

(C) 2006 American Institute of Physics. [DOI: 10.1063/1.2396925]

\section{INTRODUCTION}

In many solid-liquid flows, the particle-particle and particle-wall collisions play an important role in determining the bulk dynamics. Though the particle-on-wall immersed collision has been examined over the past two decades, ${ }^{1-8}$ the particle-on-particle collisions in a viscous liquid have not been investigated; these collisions may differ from a particlewall collision because of the mobility of the impact particle. For flows with negligible fluid effects, the inelastic and frictional particle collisions dominate the bulk behavior and dissipate most of the flow kinetic energy. With increasing effects of the interstitial liquids, the bulk changes its dispersive nature due to the lubricating fluid. If the liquid has a comparable density to the solid phase, the fluid motion and its non-negligible viscous effects introduce additional mechanisms for momentum transport and energy dissipation. To address the problem of how the interstitial liquid affects the bulk behavior, it is crucial to understand first the solid-liquid interaction at the particle-level.

Davis, Sarayssol, and $\mathrm{Hinch}^{2}$ used elastohydrodynamic theory (EHL) to examine the elastic solid deformation owing to the increase in the liquid pressure for an immersed collision between two spheres. Their work revealed the importance of particle inertia in determining the solid motion and its deformation. Upon solving the equation of motion, the particle Stokes number, $\mathrm{St}=m^{*} U_{i} / 6 \pi \mu a^{* 2}$, was defined to characterize the counterbalancing solid inertia and steady viscous drag in terms of the reduced mass $m^{*}=\left(1 / m_{1}+1 / m_{2}\right)^{-1}$, the reduced radius $a^{*}=\left(1 / a_{1}+1 / a_{2}\right)^{-1}$, relative velocity $U_{i}$ between the two spheres, and liquid viscosity $\mu$. For an immersed normal on-wall collision, ${ }^{4,5}$ the sphere rebound and impact velocities in a liquid, $U_{r}$ and $U_{i}$, are often used to define the effective coefficient of restitution, $e=-U_{r} / U_{i}$, which characterizes the total energy loss in both phases. The effective coefficient of restitution was

\footnotetext{
a) Author to whom correspondence should be addressed. Electronic mail: hunt@caltech.edu
}

found to decrease monotonically with diminishing particle Stokes number. For an impact at high particle Stokes number, St $>2000$, the fluid becomes negligible resulting in a nearly unity restitution coefficient that approximates a dry impact. However, with increasing liquid viscosity or decreasing particle inertia, the sphere can no longer sustain its motion through the liquid and a critical particle Stokes number, St $\sim 10$, exists below which no rebound occurs.

For a general immersed oblique particle-on-wall collision, experimental evidence has suggested the decomposition of the normal and the tangential components of motion. ${ }^{6,7}$ Using the surface normal as reference, Joseph and $\mathrm{Hunt}^{7}$ decomposed the sphere impact and rebound velocities at its center of mass and used the ratio of the normal velocities to define a normal effective coefficient of restitution $e_{n}=-U_{r n} / U_{i n}$. The normal particle Stokes number was also defined using the normal impact velocity. The correlation between the two parameters followed the same trend as the reported results for immersed normal on-wall collisions. The same trend was also observed by Kantak and Davis, ${ }^{6}$ who dropped a sphere obliquely onto a wall layered with a lubricant film. As for the tangential component of motion, Joseph and Hunt $^{7}$ first measured the tangential velocities, $U_{i t, c p}$ and $U_{r t, c p}$, at the contact point before and after an oblique collision. These velocities depend on both the tangential and the angular velocities of the sphere center of mass and can be calculated as $U_{i t, c p}=U_{i t}+a \omega_{i}$ and $U_{r t, c p}=U_{r t}+a \omega_{r}$ for the impact and rebound motion, respectively. The effective angles of incidence and rebound, $\Psi_{i}=U_{i t, c p} / U_{i n}$ and $\Psi_{r}=U_{r t, c p} / U_{i n}$ were used to estimate the friction coefficient $\mu_{C}$ and the rotational coefficient of restitution $\beta$ for an oblique collision. Compared with Maw et al.'s dry collision model, ${ }^{8}$ Joseph and Hunt concluded that the tangential interaction at the contact point is similar to a dry system, but with a lowered friction coefficient due to the lubrication effects. However, for collisions of rough particles at increasing tangential Stokes number, $\mathrm{St}_{t}=m^{*} U_{i t} / 6 \pi \mu a^{* 2}$, the friction coefficient rose to a value that approximates the dry friction coefficient. 
They attributed this phenomenon to an increase in liquid viscosity due to the hydrodynamic pressure.

To recapitulate, for a general immersed particle-on-wall collision, the normal component of motion can be characterized with the normal effective coefficient of restitution and the normal particle Stokes number. The tangential motion at the contact point can be described similarly to a dry system but with a lubricated friction coefficient in a dry collision model. It thus raises the question of whether an immersed particle-on-particle collision exhibits these features. When the target wall is replaced by a sphere, not only do the hydrodynamic forces change correspondingly, but the mobility of the target will further complicate the collision process. To address this problem, this work first assumes that the tangential interaction between the two colliding surfaces has negligible effects on the normal component of motion. The decomposition thus suggests separate analyses of the particle interaction in the normal and the tangential directions. The following section develops the pertinent parameters and Sec. III briefs the experimental setup. In Sec. IV, the immersed normal collision between two spheres is examined first and followed by some phenomena discovered in the experiments. The tangential component of motion is then investigated at both the contact point and the center of mass. With the existing collision models and the available immersed particle-wall collision results, the current work presents methods to predict the post-collision velocities for an immersed collision.

\section{PHYSICAL PARAMETERS}

\section{A. Effective coefficient of restitution}

When a particle-on-wall collision occurs with significant interstitial fluid effects, the effective coefficient of restitution $e=-U_{r} / U_{i}$ has been used to characterize the overall energy loss in both phases. When two spheres that move in the same direction collide at velocities $U_{i 1}$ and $U_{i 2}$ in a liquid, the effective coefficient of restitution can be calculated using their rebound velocities, $U_{r 1}$ and $U_{r 2}$, as

$$
e \equiv-\frac{\left(U_{r 1}-U_{r 2}\right)}{\left(U_{i 1}-U_{i 2}\right)},
$$

in analogy with the conventional definition for binary dry collision. For oblique collisions, the normal components of the impact and rebound velocities of each sphere are used in Eq. (1) and indicated by $e_{n}$.

\section{B. Binary Stokes number}

A collision consists of a compression followed by a restitution process. For a dry collision, Poisson's hypothesis relates the restitution and the compression impulses, $P_{r}$ and $P_{c}$, with the dry coefficient of restitution as $e_{\mathrm{dry}}=P_{r} / P_{c}{ }^{9}$. This concept can be generalized to describe an immersed interparticle collision where the hydrodynamic forces are included in the compression and restitution processes. If the total fluid force on the approaching impact sphere of mass $m_{1}$ is $h_{c 1}(t)$, the fluid impulse can be estimated by a time integral $H_{c 1}=\int_{0}^{\tau_{c}} h_{c 1}(\tau) d \tau$. The integration starts from some reference time to the moment, $\tau_{c}$, when the two spheres collide initiating the solid compression. A consecutive time integral of the mutual contact force determines the solid compression impulse $P_{c}$ that also acts on the target sphere of mass $m_{2}$. Since the two spheres could be of dissimilar sizes, shapes, and velocities, a different fluid force $h_{c 2}(t)$ determines another fluid impulse $H_{c 2}=\int_{0}^{\tau_{c}} h_{c 2}(\tau) d \tau$ on the target sphere during the approach. A set of momentum equations can be found for the generalized compression process,

$$
\begin{aligned}
& m_{1} U_{i 1}-H_{c 1}-P_{c}=m_{1} U_{G 0}, \\
& m_{2} U_{i 2}-H_{c 2}+P_{c}=m_{2} U_{G 0} .
\end{aligned}
$$

The velocity $U_{G 0}$ is the group velocity while the two particles move together with mutual contact. A similar set of equations can be derived for the generalized restitution process,

$$
\begin{aligned}
& m_{1} U_{G 0}-H_{r 1}-P_{r}=m_{1} U_{r 1}, \\
& m_{2} U_{G 0}-H_{r 2}+P_{r}=m_{2} U_{r 2},
\end{aligned}
$$

including the solid restitution impulse $P_{r}$ and hydrodynamic rebound impulses, $H_{r 1}$ and $H_{r 2}$.

Substituting Eqs. (2) and (3) into Eq. (1), the effective coefficient of restitution can be represented as

$$
e=-\frac{U_{r 1}-U_{r 2}}{U_{i 1}-U_{i 2}}=\frac{P_{r}+m^{*}\left(\Delta U_{r 1}-\Delta U_{r 2}\right)}{P_{c}+m^{*}\left(\Delta U_{i 1}-\Delta U_{i 2}\right)},
$$

with a reduced mass $m^{*}$ of the particle system. The term $\Delta U_{(i, r) k}=H_{(i, r) k} / m_{k}$ represents the velocity change of particle $k$ by the hydrodynamic impulses during the impact or rebound indicated by the subscripts $i$ and $r$. The terms $\left(\Delta U_{(i, r) 1}-\Delta U_{(i, r) 2}\right)$ thus characterize the total momentum changes in the solid pair by the fluid action. For a dry binary collision, both $\Delta U_{(i, r) 1}$ and $\Delta U_{(i, r) 2}$ are zero and Poisson's hypothesis $P_{r}=e_{\mathrm{dry}} P_{c}$ is recovered. For a particle-on-wall immersed collision, the particle mass $m_{p}$ equals $m^{*}$ and Eq. (4) becomes $e=-U_{r 1} / U_{i 1}=P_{r}+m_{p} \Delta U_{r 1} / P_{c}+m_{p} \Delta U_{i 1}=P_{r}^{*} / P_{c}^{*}$. The effective coefficient of restitution thus can be interpreted as the ratio of two generalized impulses, $P_{c}^{*}$ and $P_{r}^{*}$, that considers the additional momentum change in the particle system by the fluid forces. For a particle-wall impact at higher particle Stokes numbers, the fluid is negligible ensuring smaller $m_{p} \Delta U_{i 1}$ and $m_{p} \Delta U_{r 1}$. The resultant effective coefficient of restitution would be close to a dry value $e_{\mathrm{dry}}=P_{r} / P_{c}$.

In analogy with the single-particle Stokes number, the binary particle Stokes number for the colliding spheres follows as

$$
\mathrm{St}_{B}=\frac{m^{*}\left(U_{i 1}-U_{i 2}\right)}{6 \pi \mu a^{* 2}},
$$

in which the particle pair is characterized as a single particle of effective mass $m^{*}$ and reduced radius $a^{*}$ that moves at an approach velocity $U_{\text {rel }}=\left(U_{i 1}-U_{i 2}\right)$. The numerator provides a measure of available momentum in the solid phase that sustains the particle motion through the liquid. The denominator can be interpreted as a viscous impulse by multiplying an 


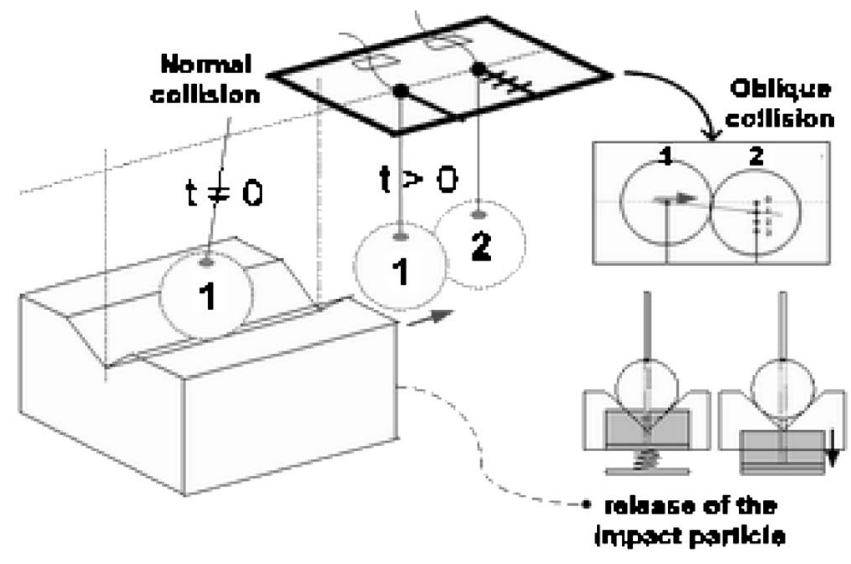

FIG. 1. Schematic experiment setup.

effective Stokes drag, $6 \pi \mu a^{*} U_{\text {rel }}$, with a forcing duration $a^{*} / U_{\text {rel }}$. The correlation between $m^{*}\left(\Delta U_{i 1}-\Delta U_{i 2}\right)$ in Eq. (4) and $m^{*}\left(U_{i 1}-U_{i 2}\right)$ in Eq. (5) implies a dependence of $e$ on $\mathrm{St}_{B}$, as observed in the particle-wall collision results. For a binary collision between spheres of identical size, as used in the current experiments, the binary Stokes number can be written as

$$
\mathrm{St}_{B}=\frac{2}{9} \frac{\rho_{p}^{*}}{\rho_{f}} \operatorname{Re}_{\mathrm{rel}},
$$

with an effective solid density $\rho_{p}^{*}=\left(1 / \rho_{1}+1 / \rho_{2}\right)^{-1}$ and the relative Reynolds number $\operatorname{Re}_{\text {rel }}=2 a \rho_{f} U_{\text {rel }} / \mu$ in accordance with the conventional definition $\mathrm{St}=\left(\rho_{p} / \rho_{f}\right) \mathrm{Re}_{i} / 9$. For oblique collisions, the normal binary Stokes number is calculated with normal relative impact velocities and denoted with $\mathrm{St}_{n}$.

\section{EXPERIMENTAL SETUP AND PROCEDURE}

As shown in Fig. 1, the particles were suspended as two pendulums in a liquid and the impact sphere was released from a V-shaped block that symmetrically supports the sphere. To control the particle orientation upon collision, a precision fixture plate was designed. Along the longitudinal plate centerline, two holes were drilled one sphere diameter apart. The plate was aligned with the V-block guiding groove to ensure an in-plane motion of the impact sphere. Thin slots were opened to the side allowing passage of the string and three consecutive holes were drilled $1.5 \mathrm{~mm}$ apart. By locating the target string in different positions, oblique collisions could be achieved.

Four types of solid spheres with $12.7 \mathrm{~mm}$ diameter were used in the experiments and their solid properties and surface roughness are summarized in Table I. The surrounding liquid was water-based glycerol solution whose viscosity is sensitive to temperature variations. Thus the liquid temperature was measured before each collision. The apparent specific weight of the solution was measured using a hydrometer to extrapolate the mixture density and viscosity from the tabulated values. In the current experiments, the apparent specific weight of the mixture measured $0-80 \%$, corresponding to a density $\rho_{f}$ between 990 and $1210 \mathrm{~kg} / \mathrm{m}^{3}$ and kinematic vis-
TABLE I. Properties of the spheres: the sphere diameter $D$, the solid density $\rho_{p}$, Young's modulus $E$, Poisson's ratio $\nu$, and the surface roughness $\sigma_{s}$ (root-mean-squared SEM measurements).

\begin{tabular}{lccccc}
\hline \hline \multicolumn{1}{c}{ Material } & $D(\mathrm{~mm})$ & $\rho_{p}\left(\mathrm{~kg} / \mathrm{m}^{3}\right)$ & $E(G P a)$ & $\nu$ & $\sigma_{\mathrm{s}}(\mu \mathrm{m})$ \\
\hline Steel ball bearing (SI) & 12.7 & 7780 & 190 & 0.27 & 0.024 \\
Steel bead (SII) & 12.7 & 7780 & 190 & 0.27 & 0.272 \\
Glass sphere & 12.7 & 2540 & 60 & 0.23 & 0.134 \\
Delrin sphere & 12.7 & 1400 & 2.8 & 0.35 & 0.796 \\
\hline \hline
\end{tabular}

cosity $\nu_{f}$ from 0.9 to $47 \times 10^{-6} \mathrm{~m}^{2} / \mathrm{s}$. The string was $0.05 \mathrm{~mm}$ nylon fishing line and its influence on the particle motion was shown to be negligible. ${ }^{7}$ Sufficient time was allowed for the motion of the target sphere and the liquid to settle between each collision.

The particle pair was illuminated from behind and the side view of a normal collision was recorded by a high speed camera at $1 \mathrm{kHz}$ frame rate. The true color (RGB) image was converted into a binary (black-white) format by properly tuning the grayness threshold, as shown from Figs. 2(a) and 2(b). A black sphere was obtained for opaque steel and Delrin spheres while a ring was generated for semitransparent glass particles. The interior of the ring was filled to represent the actual occupancy of the solid material, as shown from Figs. 2(b) and 2(c). The mean $X$ and $Y$ coordinates of the black pixels were calculated to locate the center of each sphere. The time evolution of the particle trajectory was fitted over 10 to $15 \mathrm{~ms}$ before and after the collision, as shown in Fig. 2(d), for the impact and rebound velocities $U_{i 1}, U_{r 1}$ and $U_{i 2}, U_{r 2}$ of each sphere.
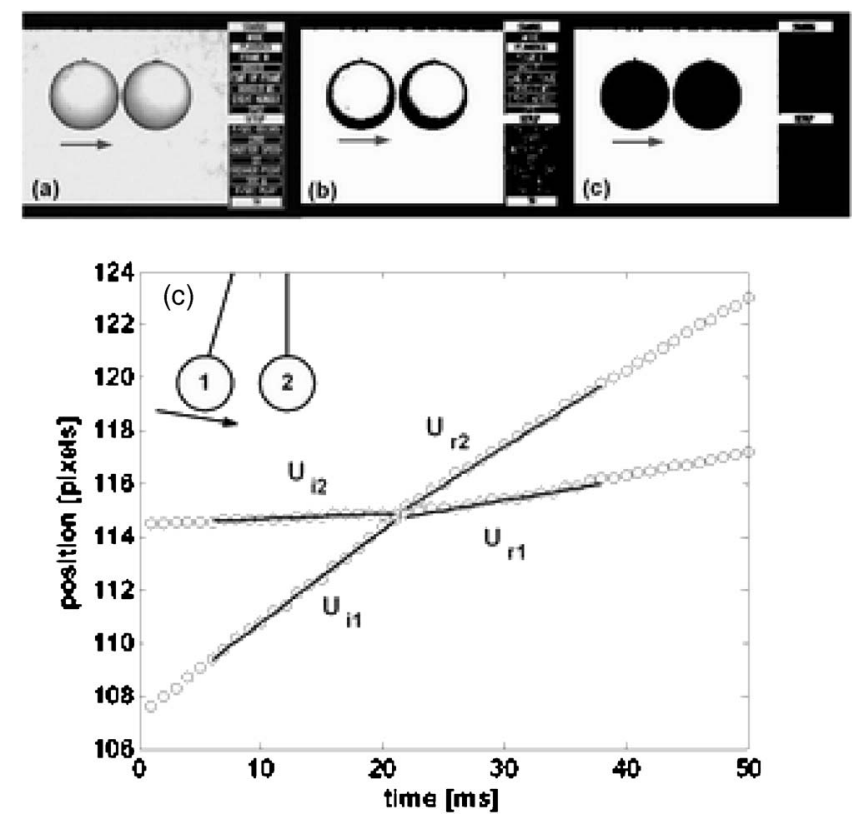

FIG. 2. A collision between two $12.7 \mathrm{~mm}$ (163 pixels) glass spheres in an aqueous solution of glycerol $(4.5 \mathrm{cP})$. The side view of the pair (a) in RGB true color, (b) after conversion into black/white binary images, and (c) after filling the interior. (d) The corresponding sphere trajectories. The sphere velocities are $U_{i 1}=27.2 \mathrm{~mm} / \mathrm{s}, U_{i 2} \approx 0.2 \mathrm{~mm} / \mathrm{s}, U_{r 1}=6.7 \mathrm{~mm} / \mathrm{s}$, and $U_{r 2}$ $=21.9 \mathrm{~mm} / \mathrm{s}$. For this collision, the effective coefficient is $e \approx 0.6$ and $\mathrm{St}_{B}$ $=20.1$. 

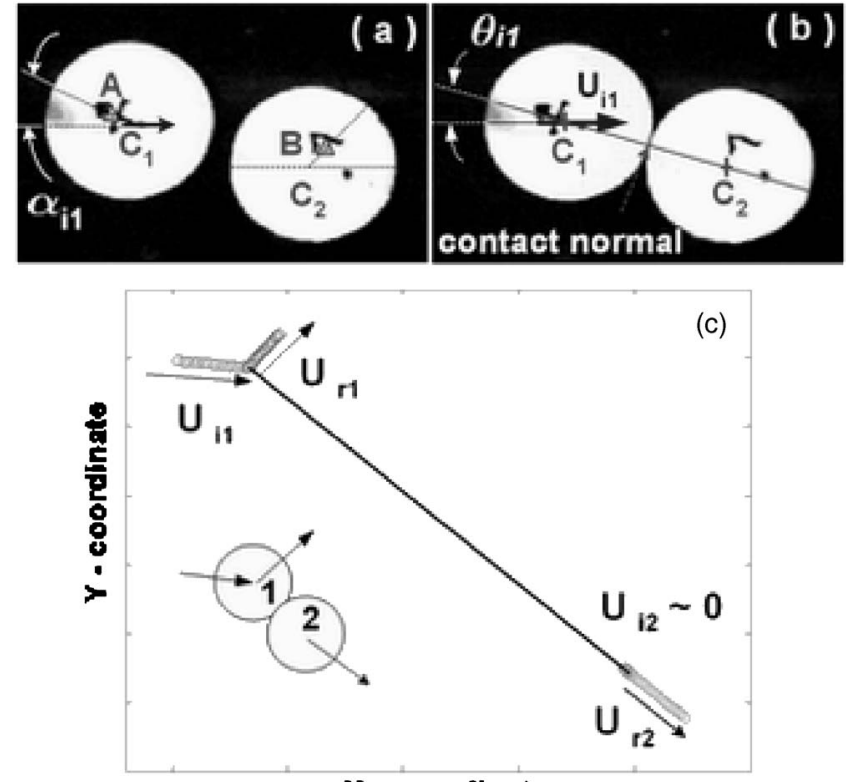

$X$ - coordinate

FIG. 3. Bottom view of an oblique collision between two Delrin spheres in water: (a) the inclination angle of the impact sphere, (b) the contact normal and the impact angle of the impact sphere, (c) time evolution of the particle trajectory. The sphere velocities are $U_{i 1}=11.94 \mathrm{~cm} / \mathrm{s}, U_{i 2} \approx 0.95 \mathrm{~cm} / \mathrm{s}$, $U_{r 1}=4.48 \mathrm{~cm} / \mathrm{s}$, and $U_{r 2}=8.24 \mathrm{~cm} / \mathrm{s}$. For this collision, the effective coefficient is $e \approx 0.39$ and $\mathrm{St}_{B}=22.3$.

As for an oblique collision, the bottom view of the sphere motion was recorded at $1 \mathrm{kHz}$. The marker is used to track the particle rotation necessitating the direct illumination of the bottom surfaces. A typical image is shown in Fig. 3(a), where the centroid of each marker is determined and indicated by the triangles $A$ and $B$. With the sphere centers of mass $C_{1}$ and $C_{2}$, the arctangent of the slope of line $\overline{A C_{1}}$ measures the inclination angle $\alpha_{i 1}$ of the impact sphere. The inclination angle for the target sphere is estimated by the same method. With the time evolution of the inclination angles, a line is fitted over $15 \mathrm{~ms}$ before the collision to estimate the angular velocities $\omega_{i 1}$. When the spheres are in contact, the line $\overline{C_{1} C_{2}}$ defines the contact normal as shown in Fig. 3(b). Prior to the collision, a second line is fitted through 15 position points of the impact sphere to define the impact angle $\theta_{i 1}$ from the contact normal. The other angles are estimated similarly. Since for most of the impact conditions investigated in this paper the target sphere remains stationary before collision, the target impact angle $\theta_{i 2}$ is set to zero unless apparent motion is observed. The translational velocities of each sphere are calculated by the distance a sphere travels from the previous frame. The mean value of 15 consecutive frames before and after collision is calculated for $U_{i 1}, U_{r 1}, U_{i 2}$, and $U_{r 2}$. A typical result depicting the trajectories of an oblique collision is shown in Fig. 3(c).

\section{RESULTS}

\section{A. Normal particle-on-particle collision}

In Fig. 4, the effective coefficient of restitution for normal collisions between identical spheres is presented as a function of the binary particle Stokes number. With dimin-

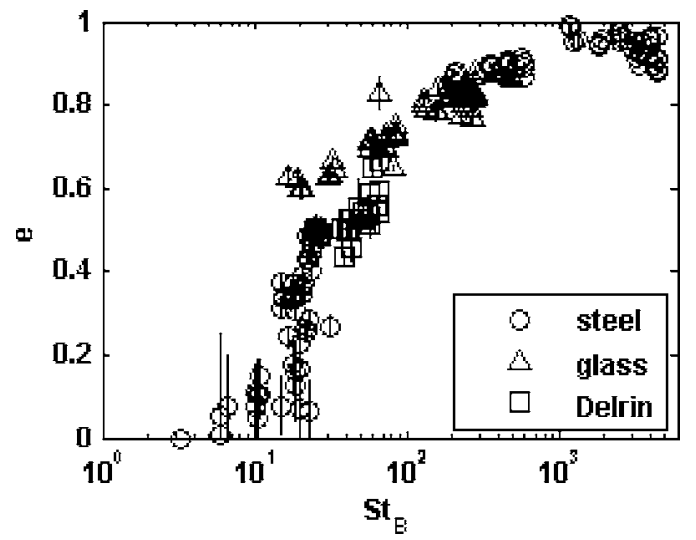

FIG. 4. Immersed normal collisions between identical spheres.

ishing $\mathrm{St}_{B}$, a monotonic decrease in $e$ is observed, a trend of which is also found for immersed normal particle-wall collisions. The error bars account for the uncertainty in estimating the sphere velocities with the least-squares line fitting. For impact at low Stokes numbers, the sphere is subject to the disturbances in the ambient flow yielding a larger error bar. Nonetheless, the general dependence of the effective coefficient of restitution on the binary Stokes number is evident.

Immersed collisions between pairs of dissimilar spheres are also investigated within the same range of liquid viscosity and impact angles. As shown in Fig. 5, a similar correlation between the effective coefficient of restitution and the binary particle Stokes number is found. In addition, when characterizing the collision by $e$ and $\mathrm{St}_{B}$, the dynamic process of a steel-on-glass impact is equivalent to a glass-onsteel event, which is also observed between the pair of steel and Delrin spheres. This phenomenon results from the use of relative velocity in both $e$ and $\mathrm{St}_{B}$. Furthermore, when the normal component of an immersed oblique interparticle collision is examined in Fig. 6, the dependence of the normal effective coefficient of restitution $e_{n}$ on the normal Stokes number $\mathrm{St}_{n}$ follows the same trend as for normal collisions. When Figs. 4-6 are compared together at high Stokes number, it is interesting to note that collisions between identical

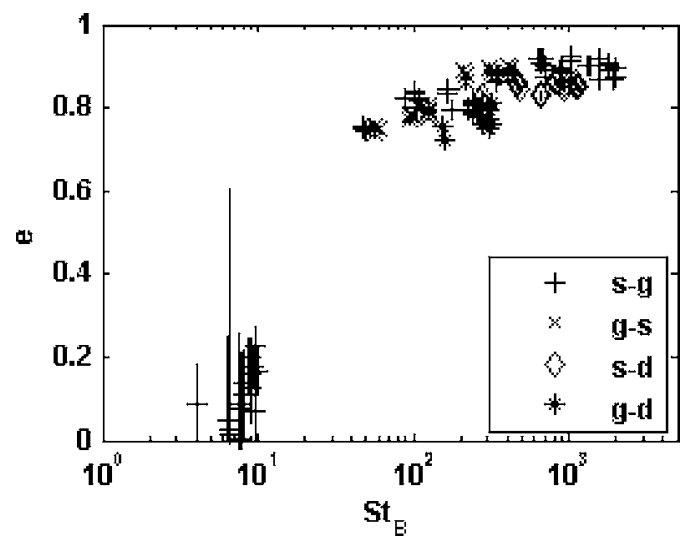

FIG. 5. Immersed normal collisions between dissimilar spheres; $s, g$, and $d$ represent steel, glass, and Delrin sphere, respectively. The first letter in the legend pair indicates the impact particle and the second for the target. 


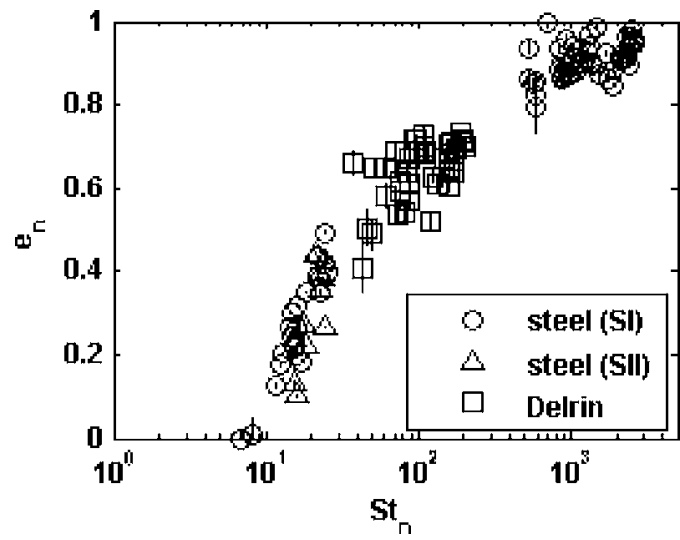

FIG. 6. Normal coefficient of restitution as a function of normal binary Stokes number for an immersed oblique collision between identical spheres.

spheres result in nearly unity restitution while dissimilar pairs consistently yield a value lower than unity.

Figure 7 compares all the current data, from both normal and oblique experiments, with the results of immersed sphere-wall normal collisions found by Joseph et al. ${ }^{4}$ General agreement between the two systems validates the definition and the usage of $e$ and $\mathrm{St}_{B}$ when characterizing the normal component of motion during an immersed collision, despite the size and mobility of the target.

Around $\mathrm{St}_{B}=15-60$, the glass-on-glass collisions result in higher restitution than the other particle pairs, which may be attributed to a large separation when two glass surfaces interact at the surface asperities. If the compression process terminates at a greater interstitial gap, the two spheres will experience smaller hydrodynamic $\mathrm{drag}^{2-4}$ guaranteeing a higher restitution. The glass spheres used in the experiments have larger surface roughness than the steel ball bearings.

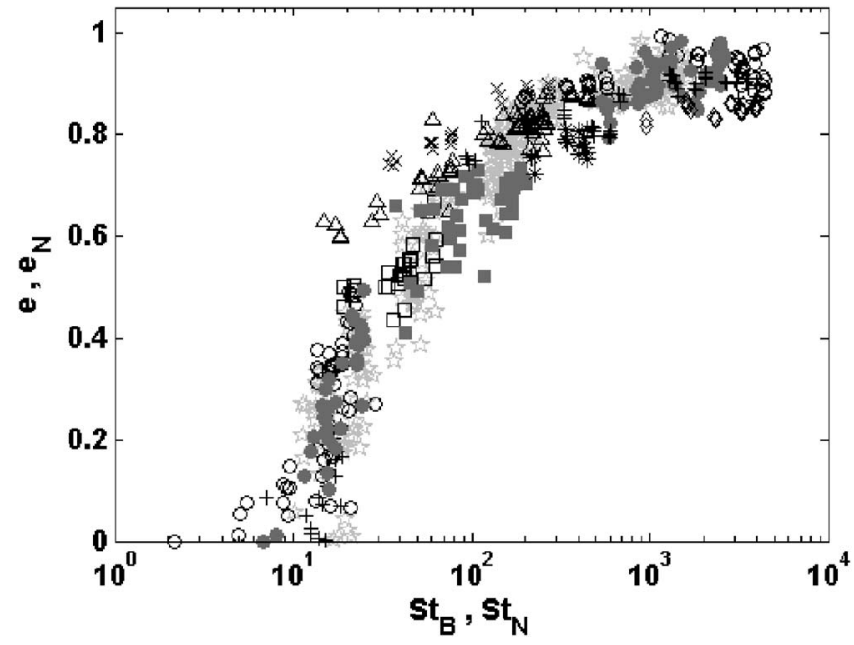

FIG. 7. The effective coefficient of restitution as a function of binary Stokes number. The interparticle collision results include normal collisions between identical pairs of steel $(\bigcirc)$, glass $(\triangle)$, and Delrin $(\square)$ spheres; dissimilar particles pairs of $s-g(+), g-s(\times), s-d(\diamond)$, and $g-d(*)$; the normal component of oblique collisions between identical pairs of steel $(\bullet)$ and Delrin (ם) spheres. The current data are compared with the on-wall measurements (沈) by Joseph et al. (Ref. 4).

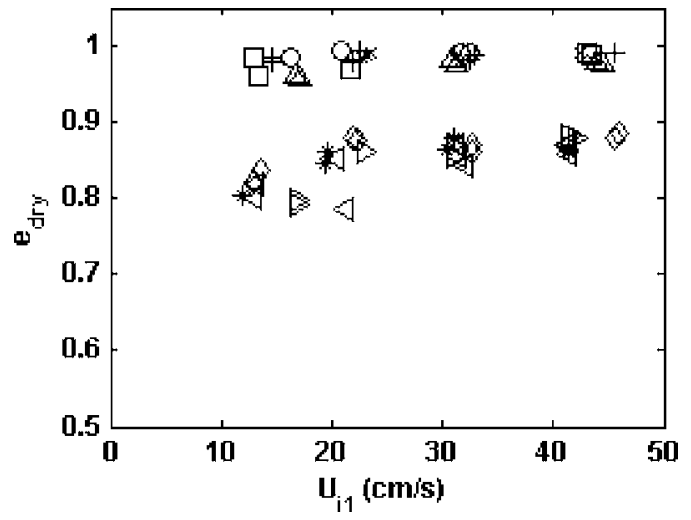

FIG. 8. Dry collision between identical pairs of steel $(\bigcirc)$, glass $(\triangle)$, and Delrin $(\square)$ spheres; dissimilar pairs of $s-g(+), g-s(\times), s-d(\diamond), d-s$ (*), $g$-d $(\triangleright)$, and $d$ - $g(\triangleleft)$.

Though the Delrin particles are rougher, their ductile asperities deform more easily than glass asperities. Consequently, the glass particles collide and rebound at a greater separation yielding higher restitution. Such asperity interactions also explain why some collisions between a steel-glass pair have higher restitution than a steel-steel pair for collisions around $\mathrm{St}_{B} \approx 10$. The enhancement, however, is less pronounced than the collisions around $\mathrm{St}_{B}=15-60$, which may result from a thicker lubrication layer ${ }^{2,3}$ that reduces the solid surface interaction. Moreover, the interparticle immersed collisions reveal zero restitution around $\mathrm{St}_{\mathrm{BC}}=3-9$; this value is slightly smaller than the critical Stokes number $\mathrm{St}_{C} \sim 10$ reported for particle-wall collisions.

To complement the current experimental results, the dry coefficient of restitution, $e_{\mathrm{dry}}$, is also measured, with zero precollision target velocity, between the same particle pairs as used in the immersed collisions (see Fig. 8). When $e_{\mathrm{dry}}$ is plotted as a function of impact velocity, the data fall in two distinct groups. For collisions between particles of similar elastic properties, a restitution coefficient close to unity is found and could be compared to the value $\overline{e_{\text {dry }}}=0.97 \pm 0.02$ reported by Joseph et $a l^{4}$ for impacts of glass and steel spheres against a glass-like Zerodur wall within the range of impact velocities $U_{i}=4-36 \mathrm{~cm} / \mathrm{s}$. The collisions between dry Delrin spheres result in $e_{\text {dry }}$ as high as between steel or glass particles. The matching elastic property diminishes the loss of kinetic energy due to internal friction or permanent deformation in the solid medium. When the Delrin particle was used with either a glass or steel sphere, plastic deformation in the softer medium may explain the observed lower $e_{\text {dry }}$. Such a decrease in the coefficient of restitution due to mismatching elastic properties in the particle pair is not observed in immersed collisions. The interstitial liquid dissipates some kinetic energy of the impact particles that diminishes the impact impulse and consequently lessens the degree of plastic deformation. Without the liquid, impacts at high velocity could induce severe plastic deformation and result in a decrease in $e_{\text {dry }}$ with increasing $U_{i}$. A considerable number of experiments have been done in this regard and are well organized in Goldsmith. ${ }^{10}$ Most of the experiments focus on impacts at high velocity, $U_{i} \geqslant 1 \mathrm{~m} / \mathrm{s}$, using either 


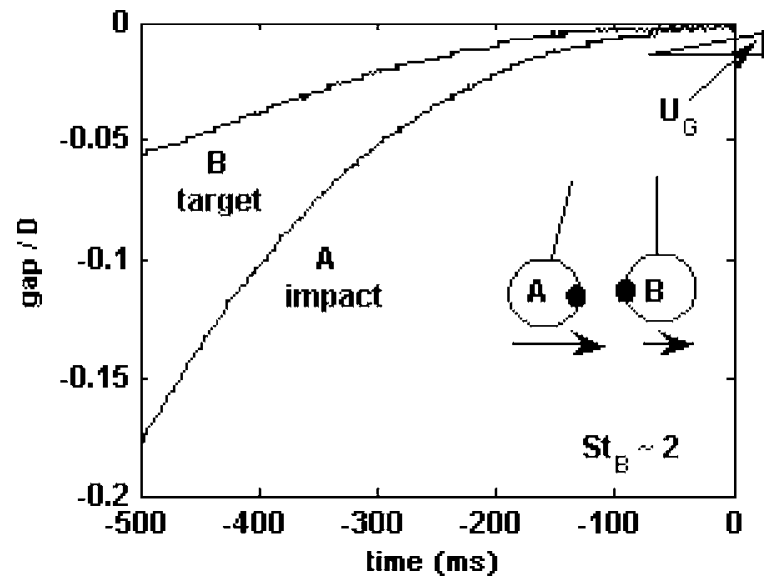

FIG. 9. Glass-on-glass immersed collision in aqueous glycerol solution $(\mu=48 \mathrm{cP})$ that results in $e \approx 0$ but $U_{G}=0.57 \mathrm{~mm} / \mathrm{s}$.

spheres of identical materials or a thick target plate of ductile metal. The current measurements thus provide new information on $e_{\mathrm{dry}}$ as a function of $U_{i}$ under different impact conditions.

\section{B. Precollision target motion and postcollision group motion}

In some immersed collisions, particularly those involving the most viscous liquid in the current experiments, the target sphere moved prior to contact. Such a precollision target motion does not occur in a dry medium. The momentum of the impact sphere is transmitted to the target by the pressure front built up in the interstitial liquid layer. As indicated in the inset of Fig. 9, two points, $A$ and $B$, can be defined one radius away from the impact and target centers to monitor the relative sphere motion. Figure 9 plots the time evolution of the particle position when a glass pair collides at $\mathrm{St}_{B}=2$. The time and the position references are set when the two spheres touch yielding $\overline{A B}=0$. The target motion is noticeable when the impact sphere is about one-fifth of a sphere diameter. Over the investigated duration of $500 \mathrm{~ms}$, the target moves about $0.07 \mathrm{D}$ yielding a nonzero velocity of $1.8 \mathrm{~mm} / \mathrm{s}$ before the two surfaces touch. The liquid is so viscous that the impact motion nearly ceases upon touching. According to the definition, the zero relative motion after collision determines a zero effective restitution while the pair is still moving at a group velocity $U_{G}=0.57 \mathrm{~mm} / \mathrm{s}$. Thus an additional parameter may be necessary to characterize the particle motion as a whole.

This precollision target motion and the grouping after impact are also observed for a steel-on-glass collision at $\mathrm{St}_{B} \sim 7$, as shown in Fig. 10(a). For a collision between two steel ball bearings at $\mathrm{St}_{B} \sim 11$, the two particles detach from each other after collision, as shown in Fig. 10(b). However, the target sphere does not accumulate sufficient inertia to advance into the liquid and the particle pair moves together at constant separation $0.02 \mathrm{D}$ after collision. As the case presented in Fig. 9, these two collisions give zero effective restitution while the particle pair is still in motion.

The precollision target motion may explain the lower
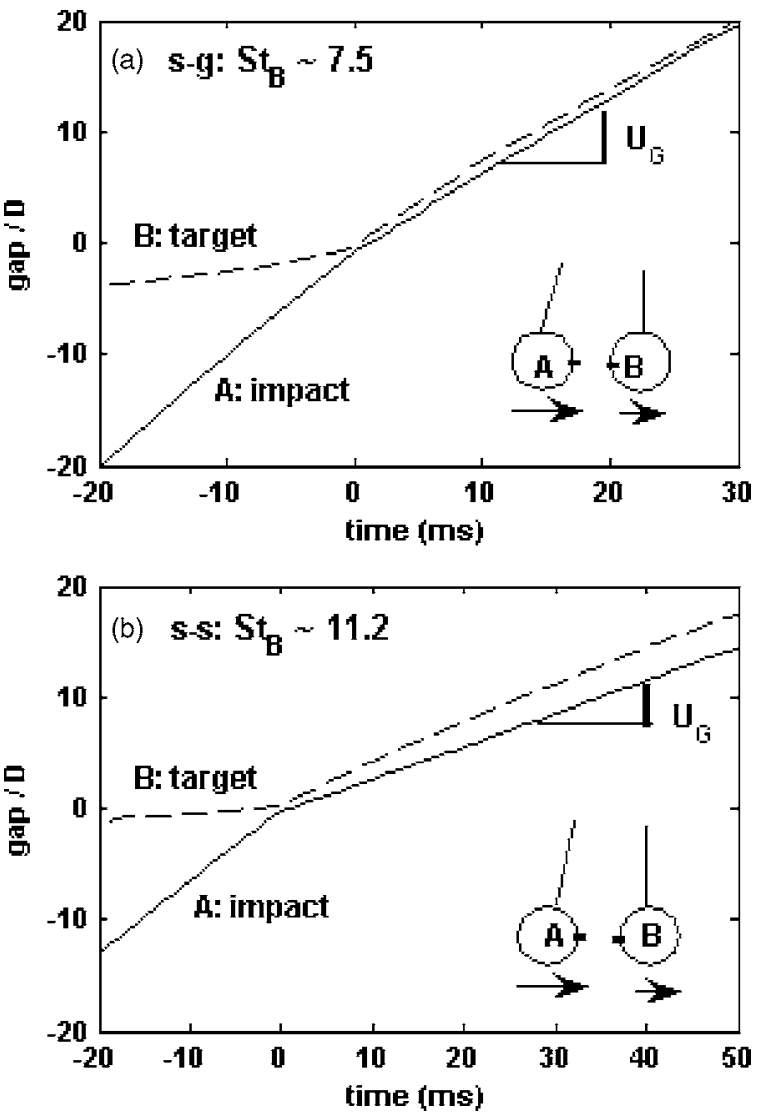

FIG. 10. Precollision target motion and postcollision group motion while zero coefficient restitution is determined for collisions between (a) steel-onglass, $U_{G} \approx 60 \mathrm{~mm} / \mathrm{s}$, and (b) steel-on-steel, $U_{G} \approx 25 \mathrm{~mm} / \mathrm{s}$, in aqueous glycerol solution $(\mu=48 \mathrm{cP})$.

critical binary Stokes numbers, $\mathrm{St}_{\mathrm{BC}}=3 \sim 9$, observed in interparticle collisions than for a particle-wall impact. The induced motion of the target particle reduces the relative velocity decreasing the fluid forces. Thus it requires in the impact sphere less inertia for rebound resulting in a smaller critical binary Stokes number. However, the effective coefficient of restitution is not sufficient to indicate the kinetic energy and the momentum left in the solid medium after collision, as illustrated by the above experiments. A new parameter is thus defined as follows and used to characterize such group motion in liquid. If the impact takes place in a dry medium, the group velocity $U_{G 0}$, at which the two spheres of mass $m_{1}$ and $m_{2}$ move in unison, is readily obtained as $U_{G 0}=m_{1} U_{i 1} /\left(m_{1}+m_{2}\right)$ using the conservation of linear momentum. By scaling the pair velocity $U_{G}$ at which the two spheres move together in liquids with $U_{G 0}$, a group efficiency number, $e_{G}$, can be defined,

$$
e_{G}=\frac{U_{G}}{U_{G 0}},
$$

as a measure of how effective the pair moves under hydrodynamic forces. The fluid effects are characterized by the binary Stokes number $\mathrm{St}_{B}$. Figure 11 presents results for experiments in which $e=0$ but $e_{G}$ is nonzero. For $\mathrm{St}_{B}<3, e_{G}$ drops from a value close to 1 ; in this regime, the viscous force dissipates most of the solid momentum and the group 


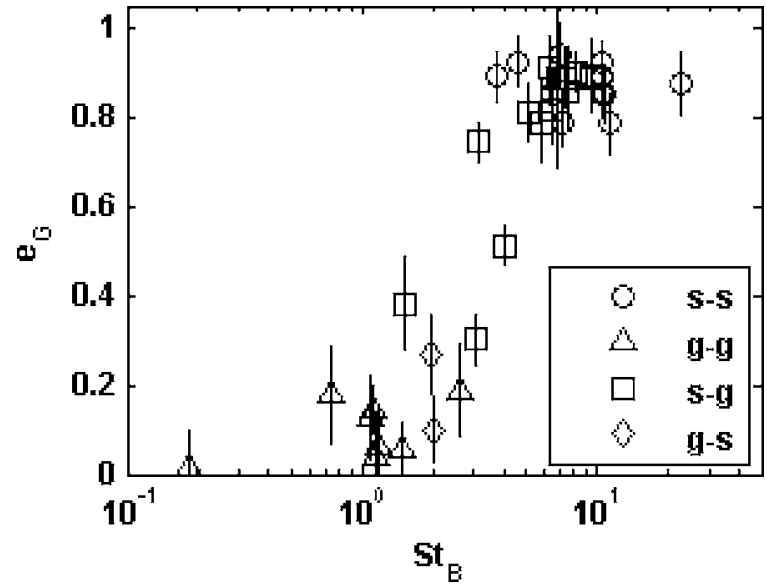

FIG. 11. Group efficiency number as a function of binary Stokes number. Error bars represent the uncertainty in estimating the sphere velocities.

motion quickly ceases after impact. For greater $\mathrm{St}_{B}$ with near unity $e_{G}$, the pair moves more effectively. The separation of the particles generates a low-pressure field in the interstitial liquid that sustains the group motion at constant gap. A critical $\mathrm{St}_{B}$ is expected beyond which the target particle would depart and break down the group motion. However, it requires further experiments to quantify this critical number. Note, the data in Fig. 7 in which $e=0$ only correspond to points in which $U_{G}$ is also equal to zero.

\section{Tangential interaction of a particle-on-particle collision}

\section{Investigation at the contact point}

The normal component of motion of an oblique interparticle collision has shown to follow the behavior of a head-on collision. This experimental finding suggests that the normal component of motion is independent of the tangential surface interaction. In order to fully describe an oblique collision, the tangential component of motion is investigated by first examining the surface interaction at the contact point using the effective angles of incidence and rebound. ${ }^{7}$ Since these effective angles correlate to Maw et al.'s nondimensional angles by a material constant, the dependence of $\boldsymbol{\Psi}_{r}=U_{r t, c p} / U_{i n}$ on $\boldsymbol{\Psi}_{i}=U_{i t, c p} / U_{i n}$ should reveal the same contact mechanism. Recall that the velocities $U_{i t, c p}$ and $U_{r t, c p}$ are measured at the contact point that consider particle rotation. Figure 12 plots the measurements for oblique immersed collisions between steel and Delrin pairs while the dashed diagonal represents a specular reflection between frictionless surfaces. Only the collisions between smooth steel ball bearings result in rebounds close to a specular reflection. The unpolished steel spheres exhibit a similar trend, deviating from specular, to the results using Delrin spheres as shown in Figs. 12(b) and 12(c). When the rough sphere impacts at $0<\boldsymbol{\Psi}_{i}$ $\leqslant 0.5$, the effective reflection angles reveal a regime that correspond to an initial sticking at the contact point. ${ }^{8}$ Negative reflection angles are observed in particle-wall collisions, which indicates reverse tangential motion of the impact particle, a motion that requires large tangential contact impulse. However, the mobility of the target sphere and the presence
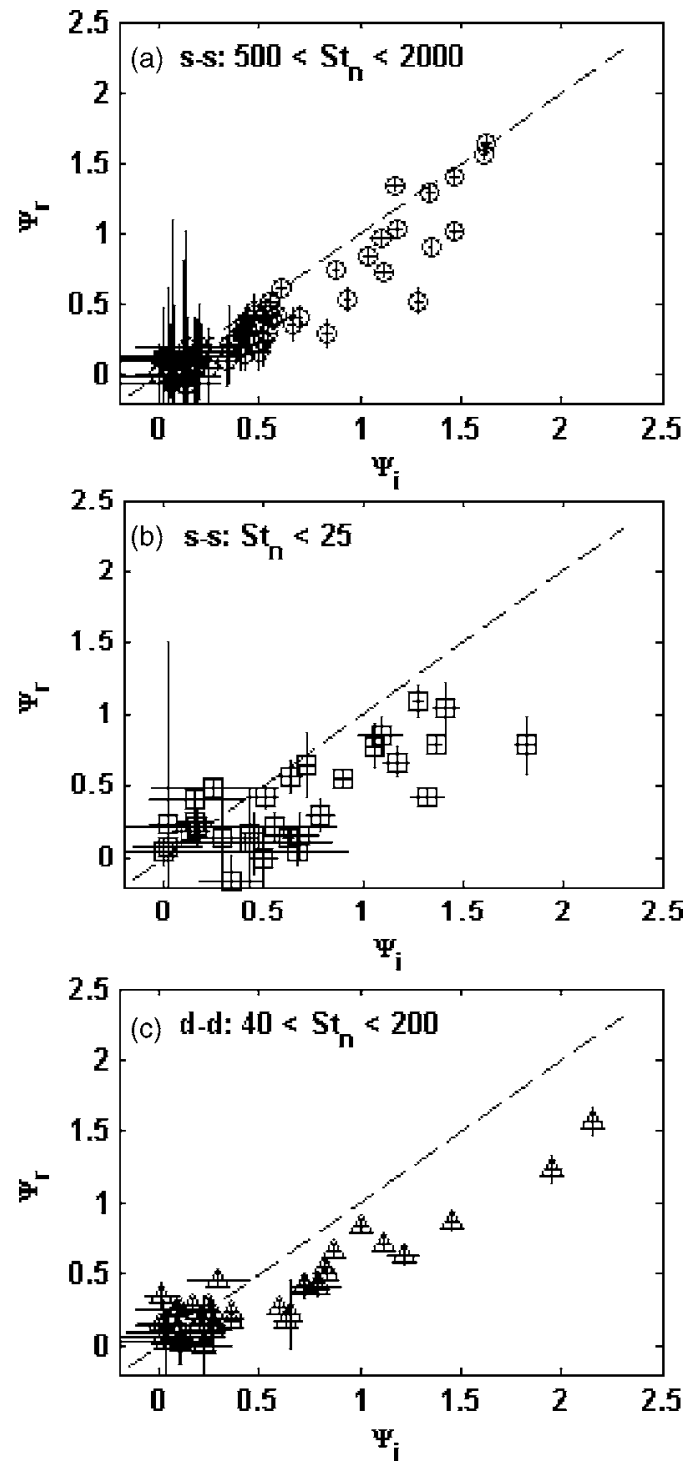

FIG. 12. The effective rebound angles, $\boldsymbol{\Psi}_{i}=U_{i t, C P} / U_{i n}$ and $\boldsymbol{\Psi} r=U_{r t, C P} / U_{i n}$, for collisions between (a) steel ball bearings, $500<\mathrm{St}_{n}<2000$, (b) unpolished steel spheres, $\mathrm{St}_{n}<25$, and (c) Delrin spheres $40<\mathrm{St}_{n}<200$.

of an interstitial liquid layer reduce both the asperity contact and the contact duration diminishing the tangential impulse. Thus a negative $\boldsymbol{\Psi}_{r}$ is rarely seen in an immersed particleparticle oblique collision.

For oblique collision against a wall, the impact takes place at nearly the same location ensuring consistent target surface condition. Joseph and $\mathrm{Hunt}^{7}$ were able to measure $\mu_{C}$ to calculate the nondimensional angles of incidence and rebound, $\psi_{i}$ and $\psi_{r}$, defined by Maw et al. ${ }^{8}$ as $\psi_{i}$ $=\left[(1-\nu) / \mu_{c}(2-\nu)\right] \boldsymbol{\Psi}_{i}$ and similarly for $\psi_{r}$ using $\boldsymbol{\Psi}_{r}$. The mobility of the target sphere may change the surface orientation and result in contact at different locations yielding more scatter in the measurements of $\mu_{C}$ between two colliding spheres. However, the data do indicate that the friction coefficient is small, approximately $\mu_{C} \leqslant 0.04$, and comparable to the value found by Joseph and Hunt ${ }^{7}$ for lubricated contacts. 


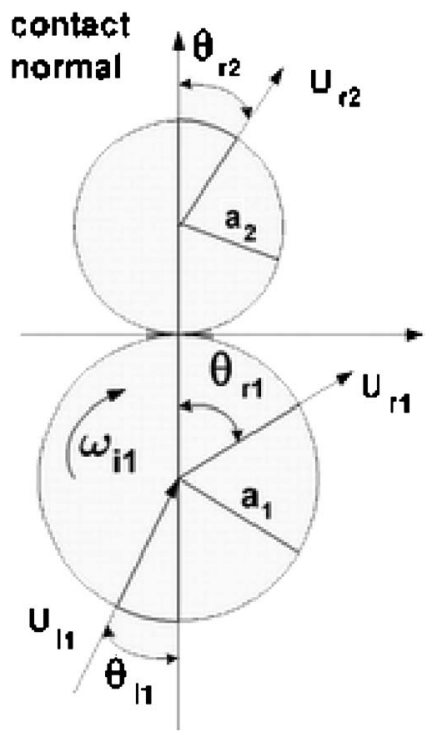

FIG. 13. Impact of two spheres in a plane motion.

\section{Investigation at the sphere centers of mass}

The contact point analysis using $\boldsymbol{\Psi}_{r}$ and $\boldsymbol{\Psi}_{i}$ investigates the tangential interaction between the colliding surfaces and can be used to determine a rotational coefficient of restitution. ${ }^{7}$ However, with the current release mechanism that diminishes the precollision particle rotation, the angular velocities remain nearly zero through the collision process, which is also a consequence of short tangential contact duration. Thus the rebound motion at the sphere centers of mass should capture the principal dynamics. A tangential collision model, following from Goldsmith, ${ }^{10}$ is presented in the following section to characterize an immersed oblique collision at the sphere center of mass. In Fig. 13, the lower sphere impacts a stationary sphere at an angle $\theta_{i 1}$ with velocities $U_{i 1}$ and $\omega_{i 1}$. The two surfaces can interact with initial sticking or sliding at the contact point and the two mechanisms rebound the spheres differently as described by Eqs. (8) and (9).

For collision with initial sticking, the normal and tangential velocities after collision can be described ${ }^{10}$ as

$$
\begin{aligned}
& U_{r 1 n}=\frac{M-e}{M+1} U_{i 1 n}, \\
& U_{r 2 n}=\frac{M}{M+1}(1+e) U_{i 1 n}, \\
& U_{r 1 t}=\frac{M+5 / 7}{M+1} U_{i 1 t}-\frac{2}{7} \frac{a \omega_{i 1}}{M+1}, \\
& U_{r 2 t}=\frac{2}{7} \frac{M}{M+1}\left(U_{i 1 t}+a \omega_{i 1}\right) .
\end{aligned}
$$

The rebound motion depends on the normal impact velocity $U_{i 1 n}$, the impact angular velocity $\omega_{i 1}$, and the mass ratio $M=m_{1} / m_{2}$. The quantity $e$ is the dry normal coefficient of restitution. The tangents of the rebound angle for both spheres can then be found as

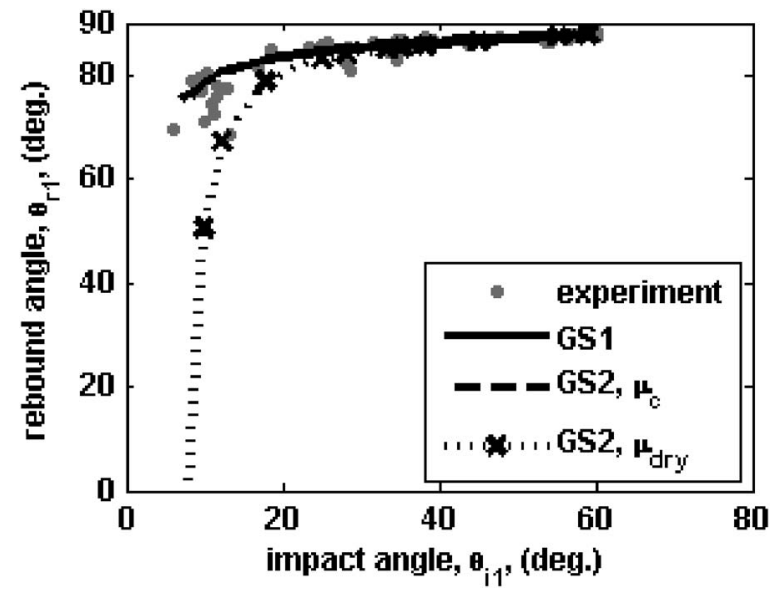

FIG. 14. The experimental rebound angle of the impact sphere is compared with the prediction from the model. Steel ball bearings in water and 500 $<\mathrm{St}_{n}<2400$. For this range of $\mathrm{St}_{n}, \overline{e_{n}}=-0.0012 \theta_{i 1}+0.9474$ with a standard deviation of $3.63 \%$.

$$
\begin{aligned}
& \tan \theta_{r 1}=\frac{M+5 / 7}{M-e} \tan \theta_{i 1}-\frac{2}{7(M-e)} \frac{a_{1} \omega_{i 1}}{U_{i 1 n}}, \\
& \tan \theta_{r 2}=\frac{2}{7(1+e)}\left[\tan \theta_{i 1}+\frac{a_{1} \omega_{i 1}}{U_{i 1 n}}\right],
\end{aligned}
$$

depending also on the impact angle $\theta_{i 1}$. Equation (8) will be indicated as the GS1 contact model.

If the collision occurs at a greater impact angle, the sphere surfaces may slide on each other initiating an initial motion that forms a second group of oblique collision referred to as GS2 contact. It can be shown that the normal rebound velocities, $U_{r 1 n}$ and $U_{r 2 n}$, are the same in both contact models. However, the friction force changes the tangential velocities and thus modifies the rebound into

$$
\begin{aligned}
& U_{r 1 t}=U_{i 1 t}-\mu_{C} \frac{1+e}{M+1} U_{i 1 n}, \\
& U_{r 2 t}=\frac{M}{M+1} \mu_{C}(1+e) U_{i 1 n}, \\
& \tan \theta_{r 1}=\frac{M+1}{M-e} \tan \theta_{i 1}-\mu_{C} \frac{1+e}{M-e},
\end{aligned}
$$

$\tan \theta_{r 2}=\mu_{C}$

involving a friction coefficient $\mu_{C}$, a value that may be modified by the interstitial liquid. Joseph and Hunt ${ }^{7}$ measured a dry friction coefficient $\mu_{\mathrm{dry}}=0.11$ for collisions between the Zerodur wall and the same steel ball bearing as used in the current experiments. With interstitial liquid, the friction coefficient drops to $\mu_{C} \sim 0.02$.

In Figs. 14-16, the two contact models are examined in predicting the rebound angle of the impact sphere. An empirical expression is fitted in the least-square sense for $e_{n}$ as a linear function of impact angle $\theta_{i 1}$ in each range of $\mathrm{St}_{n}$. For an oblique collision between two steel ball bearings at 500 $<\mathrm{St}_{n}<2400$, a general agreement between the actual re- 


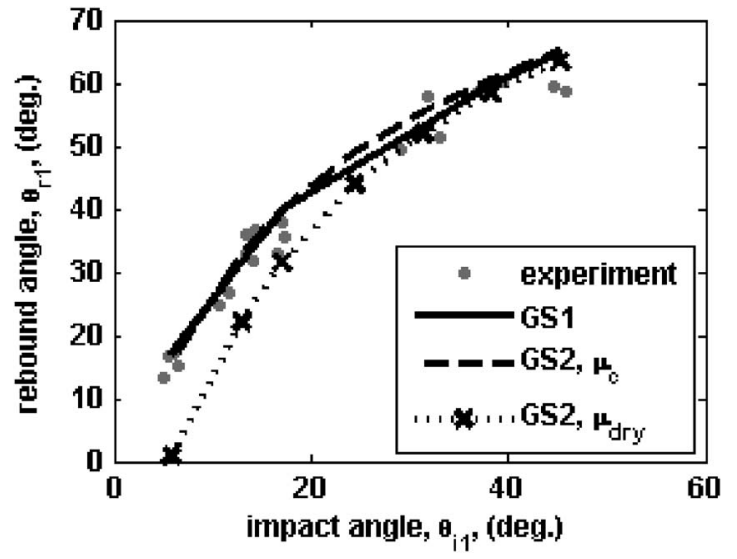

FIG. 15. Comparison between the model prediction and the experimental rebound angle. For collisions of steel ball bearings at $\mathrm{St}_{n}<25$ in aqueous glycerol solution $(48 \mathrm{cP})$, the fitted expression is $\overline{e_{n}}=-0.0081 \theta_{i 1}+0.4319$ with a standard deviation of $7.5 \%$.

bound angle and the $e_{n}-G S 1$ model prediction is observed in Fig. 14. The GS2 contact model is also examined using $e_{n}$ with both the dry and lubricated friction coefficients, $\mu_{C}=0.02$ and $\mu_{\mathrm{dry}}=0.11$. The $e_{n}-\mu_{C}-G S 2$ model captures the drop of $\theta_{r 1}$ when $\theta_{i 1}<30^{\circ}$ as well as the $e_{n}-G S 1$ model. However, using $\mu_{\text {dry }}$ in $G S 2$ results in a smaller rebound angle due to an overestimated surface friction, which suggests the non-negligible lubricating effects of the liquid when $\theta_{i 1}$ drops below $30^{\circ}$.

If the ball bearing collides at $\mathrm{St}_{n}<25$, the discrepancy between the measurements and the predictions using $e_{\text {dry }}$ and $\mu_{\mathrm{dry}}$ in GS2 is more pronounced as shown in Fig. 15. A high $e_{\text {dry }}$ requires sufficient elastic surface deformation for large restitution impulse, the deformation of which seldom occurs for impact with small inertia. Furthermore, the interstitial liquid lubricates the surfaces diminishing the physical contact. Thus using $\mu_{\text {dry }}$ in GS2 would overestimate the surface interaction and results in too small a rebound angle. When compared with the nearly constant rebound angle in Fig. 14 for collisions at high $\mathrm{St}_{n}, \theta_{r 1}$ decreases with impact angle for collisions at $\mathrm{St}_{n}<25$. Due to the lack of particle inertia, the interstitial liquid motion may synchronize the surface motion yielding a smooth decline. When $\theta_{i 1}$ is small, the slow tangential component of motion is further decelerated by the shearing lubrication force, which increases the tangential contact duration yielding a smaller $\theta_{r 1}$.

The agreement between the measurements and the model predictions supports the application of $e_{n}-G S 1$ or $e_{n}-\mu_{C}-G S 2$ contact models to describe the postcollision tangential motion. In light of the different contact mechanisms considered in $G S 1$ and $G S 2$, the current findings imply a lubricated contact that relaxes the dependence of rebound motion on the detailed tangential surface interaction. Since the physical asperity contact is diminished by the interstitial liquid, hydrodynamic effects should dominate the collision dynamics, the effects of which are realized on the effective coefficient of restitution $e_{n}$ and the lubricated friction coefficient $\mu_{C}$. The significance of a properly estimated model constant can be illustrated better in Fig. 16 when the rebound angle of a Delrin sphere is investigated over $40<\mathrm{St}_{n}<200$.

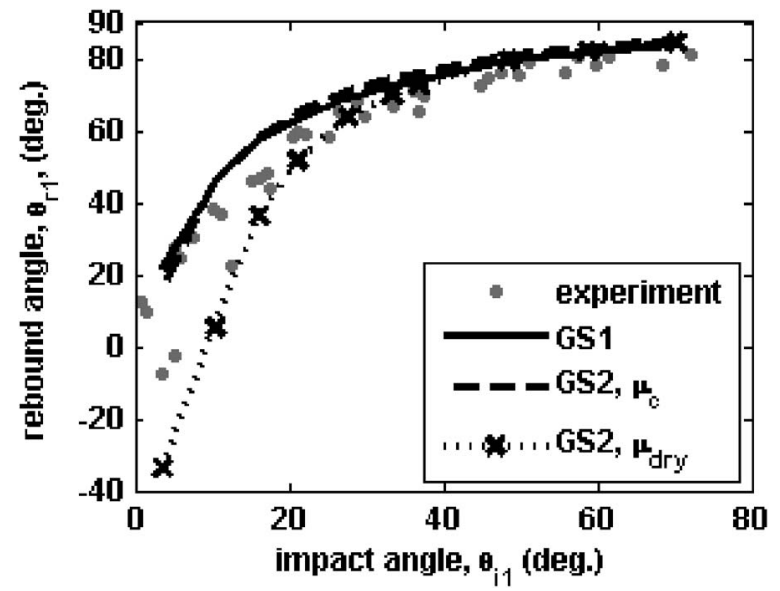

FIG. 16. The experimental rebound angle of the impact sphere is compared with the prediction from the model. Delrin spheres in water and $40<\mathrm{St}_{n}$ $<200$. For $\theta_{i 1}<50^{\circ}, \overline{e_{n}}=-0.0014 \theta_{i 1}+0.687$ while $\overline{e_{n}}=-0.0066 \theta_{i 1}+0.961$ if $\theta_{i 1} \geqslant 50^{\circ}$. The standard deviation for each fitting is $5.1 \%$ and $8.0 \%$, respectively.

For the Delrin pair, the manufacturer specifies a dynamic dry friction coefficient $\mu_{\mathrm{dry}}=0.2$. The lubricated friction coefficient, $\mu_{C}=0.1$, is approximated by the value measured between lubricated nylon rollers. As the previous two cases, the $e_{n}-G S 1$ collision model agrees well with the actual measurements. The $e_{n}-\mu_{C}-G S 2$ prediction deviates from the experiment data when $\theta_{i 1}<10^{\circ}$. The diminishing particle inertia explains the decrease in $\theta_{r 1}$ as for the steel ball bearing collision at $\mathrm{St}_{n}<25$. A slightly higher rebound angle is observed when comparing Figs. 15 and 16. Such a phenomenon can be attributed to both higher particle inertia and more asperity interaction in the tangential direction for a rougher Delrin surface. Using $\mu_{\mathrm{dry}}$ results in erroneous prediction including a reverse rebound motion that seldom occurs in the current experiments.

Using a proper $e_{n}$ in the $G S 1$ contact model predicts the rebound angle for the impact sphere for most of the investigated impact conditions. With the measured $e_{n}$, the $e_{n}-G S 1$ contact model is further examined in predicting the postcollision tangential velocity for both the impact and target spheres. As shown in Fig. 17(a), the rebound of the impact sphere is reproduced with the model. However, the target rebounds at a velocity about one order of magnitude smaller than $U_{r 1 t}$ and the data are more scattered, as shown in Fig. 17(b). The discrepancy may be attributed to the precollision motion while a stationary target is assumed in the collision model. A smaller relative velocity reduces the tangential impulse yielding a slower postcollision target motion. Rebounds faster than the model prediction, those above the solid line, are found for collisions between a Delrin pair and also for steel ball bearings impacts at $\mathrm{St}_{n}>500$. With greater asperity contact, either due to a rougher Delrin surface or enhanced by collisions with higher particle inertia, the tangential contact impulse would be higher, yielding a faster target rebound velocity.

In Fig. 18, the rebound velocity of the target steel ball bearing is compared with the prediction using GS2 with a friction coefficient higher than $\mu_{C}=0.02$. The value 

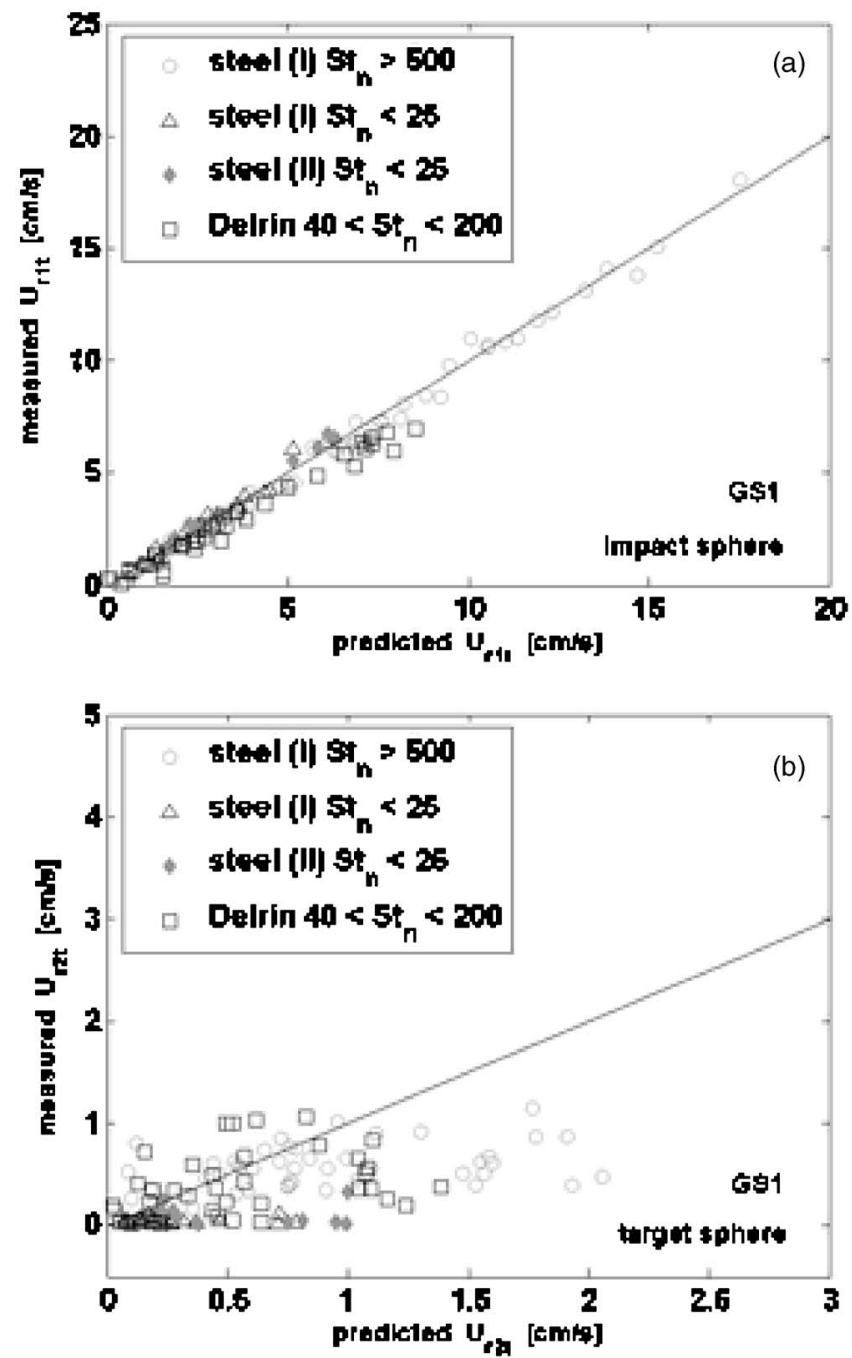

FIG. 17. Comparison of the predicted postcollision tangential velocity of (a) the impact sphere and (b) the target sphere. Steel ball bearings and the unpolished steel spheres are indicated by (I) and (II), respectively, and the solid line plots $y=x$.

$\mu_{C}=0.02$ is measured between a steel ball bearing and a Zerodur wall, ${ }^{7}$ a target of which has a smoother surface $\left(\sigma_{s}=0.0155 \mu \mathrm{m}\right)$ than the current ball bearing target. Thus using a greater friction coefficient, $\mu_{C}=0.1$, improves the model prediction, which emphasizes again the significance of a model constant that includes the liquid effects. However, a measurement of lubricated $\mu_{C}$ is usually difficult for a rapid collision process and especially prone to errors with an unconstrained target. Hence the $e_{n}-G S 1$ model is proposed to be a more practical model than the $e_{n}-\mu_{C}-G S 2$ contact model that requires an accurate $\mu_{C}$.

As the last attempt to quantify the hydrodynamic effects on an immersed interparticle oblique collision, the angle between the particle rebound trajectories is examined. If the surfaces of two colliding objects are perfectly smooth, their trajectories after a dry collision will form a $90^{\circ}$ angle. An effective rebound angle could be defined,

$$
\Pi_{r b d}=\frac{\left|\theta_{r 2}-\theta_{r 1}\right|}{90},
$$

as the ratio between the measured angle, $\left|\theta_{r 2}-\theta_{r 1}\right|$, and the dry frictionless rebound angle. A small $\Pi_{r b d}$ indicates strong

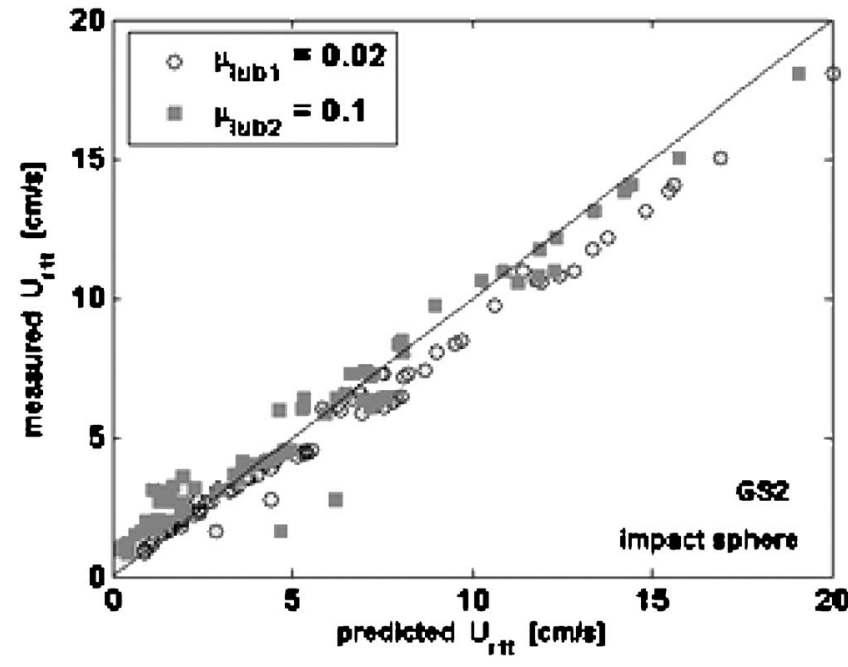

FIG. 18. GS2 prediction with a higher lubricated friction coefficient.

lubricating force that dissipates the total tangential momentum in the solid phase. To characterize this fluid effect, a tangential binary Stokes number is defined as

$$
\mathrm{St}_{t}=\frac{m^{*}\left|U_{i 1 t}-U_{i 2 t}\right|}{6 \pi \mu a^{* 2}}
$$

using the relative tangential velocity at the sphere centers. The result is plotted in Fig. 19, where a nearly $90^{\circ}$ included angle, corresponding to $\Pi_{r b d} \sim 1$, is observed at high $\mathrm{St}_{t}$, indicating negligible fluid effects. The larger tangential impact inertia also lessens the influence of physical asperity contact on particle rebound motion. Thus a rough particle colliding with small inertia, like Delrin impacts at $\mathrm{St}_{t}<100$, would rebound more randomly, a mechanism that may explain the scatter of Delrin data in Fig. 19.

\section{SUMMARY}

This paper investigates the immersed interparticle collision between spheres of identical size and identical or dissimilar materials. During contact, the line joining the sphere centers defines the contact normal upon which a collision is

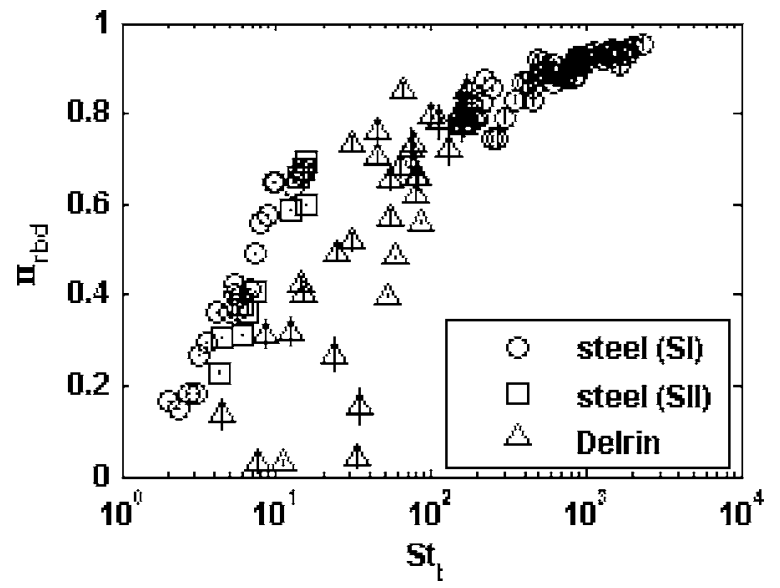

FIG. 19. The effective rebound angle as a function of tangential Stokes number. 
decomposed into a normal and a tangential component. Hence, similar to a dry binary collision, an immersed interparticle collision can be described separately in these two components of motion.

Poisson's impulse hypothesis that relates the compression and restitution process with the dry coefficient of restitution is generalized to include the hydrodynamic impulses. An effective coefficient of restitution is defined accordingly for the normal component of motion of a general immersed interparticle collision. The binary Stokes number is defined to characterize the ability of a particle pair in sustaining its motion in a viscous liquid. Since the effective coefficient of restitution characterizes the total energy loss during the collision, it decreases with diminishing binary Stokes number. Despite the size and the mobility of the target, the current experiments reveal the same correlation between these two parameters as the one observed for an on-wall collision. For an oblique interparticle collision, the two parameters are modified using the normal components of motion. The agreement with the normal collision data confirms the usage of $e_{n}$ and $\mathrm{St}_{n}$. This experimental finding also supports the hypothesis that the normal component of motion in an oblique collision is not affected by the tangential surface interaction.

In general, the postcollision tangential motion can be described by a collision model as long as the model parameters, such as the friction coefficient and the normal coefficient of restitution, are properly modified for the hydrodynamic effects. In addition to the surface properties, a lubricated friction coefficient is also sensitive to the impact condition and usually is difficult to measure. Thus Goldsmith's second contact model that involves the friction coefficient is of less practical value. Fortunately, the prediction with Goldsmith's first model, requiring only the effective normal coefficient of restitution, agrees with the actual sphere motion at its center of mass. Thus the $e_{n}-G S 1$ model is proposed to describe the tangential motion of an immersed collision.
The tangential motion at the contact point is also compared with Maw et al.'s theory, ${ }^{8}$ which describes the dry surface tangential interaction. The mobility of the target sphere and the interstitial liquid reduces the tangential impulse upon collision. With higher effective incidence angle, a sliding mechanism better describes the experimental findings, and an impact at lower incidence angle may be described by a sticking-then-sliding process. Interesting phenomena, such as precollision target motion and postcollision group velocity of a particle pair, are also observed. The target mobility and the incompressible surrounding liquid are essential for such observations, which have not been reported in on-wall immersed collision experiments and cannot occur in a dry medium.

\section{ACKNOWLEDGMENTS}

This work was supported by National Science Foundation Grant No. CTS-0314005. We are grateful to G. G. Joseph for sharing his data, and we acknowledge H.-Y. Chiu for the SEM measurements of our steel beads (SII).

\footnotetext{
${ }^{1}$ M. H. McLaughlin, "An experimental study of particle-wall collision relating to flow of solid particles in a fluid," Engineer's degree thesis, California Institute of Technology, Pasadena, California (1968).

${ }^{2}$ R. H. Davis, J.-M. Serayssol, and E. J. Hinch, "The elastohydrodynamic collision of two spheres," J. Fluid Mech. 163, 479 (1986).

${ }^{3}$ G. Barnocky and R. H. Davis, "Elastohydrodynamic collision and rebound of spheres: Experimental verification," Phys. Fluids 31, 1324 (1988).

${ }^{4}$ G. G. Joseph, R. Zenit, M. L. Hunt, and A. M. Rosenwinkel, "Particlewall collisions in a viscous fluid," J. Fluid Mech. 433, 329 (2001).

${ }^{5}$ P. M. Gondret, M. Lance, and L. Petit, "Bouncing motion of spherical particles in fluids," Phys. Fluids 14, 643 (2002).

${ }^{6}$ A. A. Kantak and R. H. Davis, "Oblique collisions and rebound of spheres from a wetted surface," J. Fluid Mech. 509, 63 (2004).

${ }^{7}$ G. G. Joseph and M. L. Hunt, "Oblique particle-wall collisions in a liquid," J. Fluid Mech. 510, 71 (2004).

${ }^{8}$ N. Maw, J. R. Barber, and J. N. Fawcett, "The role of elastic tangential compliance in oblique impact," J. Lubr. Technol. 103, 74 (1981).

${ }^{9}$ W. J. Stronge, "Rigid body collisions with friction," Proc. R. Soc. London, Ser. A 431, 169 (1990).

${ }^{10}$ W. Goldsmith, Impact (Dover, New York, 2001).
} 